\title{
Integral Formulation of the Measured Equation of Invariance: A Novel Sparse Matrix Boundary Element Method
}

\author{
Juan M. Rius, Rafael Pous and Angel Cardama \\ Dpt. Signal Theory and Communications, Universitat Politecnica de Catalunya, Barcelona, Spain
}

\begin{abstract}
A novel integral formulation of the Measured Equation of Invariance method is derived from the reciprocity theorem. This formulation uses the electric and magnetic Green's functions of the environment to obtain a matrix equation for the induced surface current with the same number of unknowns as the conventional Boundary Element - Method of Moments (BE-MoM) approach. However, the matrix that must be inverted in the new formulation is sparse and circulant, with only three non-zero elements per row. Sample results for two-dimensional TM and TE problems with perfectly conducting scatterers show enormous CPU time and memory savings over the conventional BEM-MoM approach. The new formulation has important advantages over the original finite difference formulation of MEI, but also shares some of its limitations.
\end{abstract}

\section{INTRODUCTION}

Surface integral equation (boundary element) methods are widely used in conjunction with the method of moments in the numerical solution of electromagnetic radiation and scattering problems [1] [2]. In contrast to finite difference (FD) and finite element (FE) methods, which require a volumetric meshing of the space and some mesh termination conditions [3], the surface integral equation methods enforce the boundary conditions only on the interfaces between homogeneous regions, and thus require only a surface meshing of the boundaries. This results in a much smaller number of unknowns than in the FD or FE methods.

On the other hand, the FD and FE methods have the advantage of producing a sparse matrix, while the classical formulation of the integral equation methods [1] obtains a full matrix. The sparse matrix of the finite methods requires a drastically smaller computational burden to be inverted, but - unless the mesh is truncated near to the object - usually the substantially larger number of unknowns outweights the computational advantage. In or-

Manuscript received July 10, 1995

J.M.Rius, e-mail rius@voltor.upc.es, fax +34-3-4017232

This work has been supported by the Spanish 'Comisión Interministerial de Ciencia y Tecnologia' (CICYT) under the project: TIC 93-0518. der to reduce the number of unknowns, some recent work has been directed towards terminating the mesh as close as possible to the scatterer [4] [5]. However, those radiation or absorbing boundary conditions are not robust. A rigorous mesh termination can be achieved in a hybrid finite element - boundary element method (FE-BEM) [6]. However, this technique still requires the inversion of a full matrix associated with the mesh boundary.

Recently, the Measured Equation of Invariance (MEI) method has been introduced by Mei et al. [7] and Pous [8] as a mesh truncation condition for the FD method, which allows the termination of the mesh very close to the scatterer surface. This approach has proved to be more robust than the absorbing or radiation boundary conditions applied close to the scatterer surface and, in contrast to the hybrid FE-BEM method, the interrelation between the field samples on the boundary is sparse.

In this paper we present a new concept of the MEI as a surface integral equation method derived from the reciprocity theorem, which produces a sparse matrix with the same number of unknowns as the conventional BE-MoM technique [9]. It will be shown that the mesh truncation condition of Mei et al. [7] [8] is in fact a hybrid FD-BEM approach in which a surface integral equation formulation is used to terminate the finite difference mesh.

\section{INTEgRAL EQUATION FORMULATION OF THE MEI}

\section{A. Derivation from the reciprocity theorem}

Reciprocity theorem states that two sets of electric and magnetic sources $\left(\vec{J}_{1}, \vec{M}_{1}\right)$ and $\left(\vec{J}_{2}, \vec{M}_{2}\right)$ that radiate simultaneously at the same frequency in a linear and isotropic medium, respectively produce electric and magnetic fields $\left(\vec{E}_{1}, \vec{H}_{1}\right)$ and $\left(\vec{E}_{2}, \vec{H}_{2}\right)$ that satisfy:

$$
\int_{V}\left(\vec{E}_{1} \cdot \vec{J}_{2}-\vec{H}_{1} \cdot \vec{M}_{2}\right) d v=\int_{V}\left(\vec{E}_{2} \cdot \vec{J}_{1}-\vec{H}_{2} \cdot \vec{M}_{1}\right) d v
$$

Let $C$ be the boundary of a perfectly conducting (PEC) scatterer and $C_{0}$ a portion of this boundary (Figure 1). Let the first set of electric and magnetic sources be the induced currents on the surface of the PEC scatterer $\vec{J}_{1}=$ $\hat{n} \times\left.\vec{H}\right|_{C}=\overrightarrow{J_{s}}$ and $\vec{M}_{1}=-\hat{n} \times\left.\vec{E}\right|_{C}=0$, where $\vec{E}$ and 


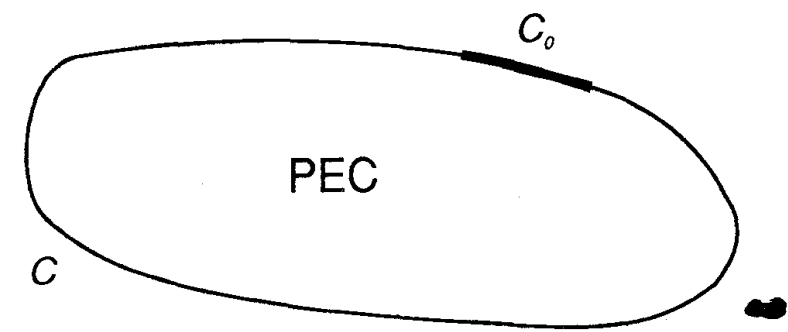

Fig. 1. Boundary of a perfectly conducting (PEC) scatterer, and a portion of this boundary.

$\vec{H}$ are the electric and magnetic fields actually existing in the presence of the scatterer. According to the equivalence theorem, the induced currents on a PEC surface are equal to the surface equivalent currents that radiate in free space the scattered fields $\vec{E}^{s}=\vec{E}-\vec{E}^{i}, \vec{H}^{s}=\vec{H}-\vec{H}^{i}$, where $\vec{E}^{i}$ and $\vec{H}^{i}$ are the fields in the absence of the scatterer, or the incident fields. Therefore, in equation (1) we have $\vec{J}_{1}=\vec{J}_{s}, \vec{M}_{1}=0, \vec{E}_{1}=\vec{E}^{s}, \vec{H}_{1}=\vec{H}^{s}$. Let the second set of electric and magnetic sources $\left(\vec{J}_{2}, \vec{M}_{2}\right)$ exist only over $C_{0}$ and radiate electric field $\vec{E}_{2}=\vec{E}_{\text {null }}$. With these sets of sources, equation (1) becomes:

$$
\int_{C_{0}}\left(\vec{E}^{s} \cdot \vec{J}_{2}-\vec{H}^{s} \cdot \vec{M}_{2}\right) d \ell=\int_{C} \vec{E}_{\text {null }} \cdot \vec{J}_{s} d \ell=\left\langle\vec{E}_{\text {null }}^{*}, \vec{J}_{s}\right\rangle
$$

where $\langle f, g\rangle$ is the Hilbert inner product defined along the scatterer boundary $C$. Since $\vec{J}_{2}$ and $\vec{M}_{2}$ represent surface currents, they are vectors tangent to the scatterer boundary and therefore:

$$
\int_{C_{0}}\left(\vec{E}_{t}^{s} \cdot \vec{J}_{2}-\vec{H}_{t}^{s} \cdot \vec{M}_{2}\right) d \ell=\left\langle\vec{E}_{\text {null }}^{*}, \vec{J}_{s}\right\rangle
$$

where $\vec{E}_{t}^{s}$ and $\vec{H}_{t}^{s}$ are the components of the scattered fields tangential to the scatterer boundary and $\vec{E}_{\text {null }}$ is the field radiated by the set of currents $\vec{J}_{2}$ and $\vec{M}_{2}$ that exist along $C_{0}$. Equation (3) is the integral equation formulation of the MEI (IE-MEI).

\section{B. Discretization of the IE-MEI for 2-D problems}

For simplicity, only the discretization for 2-D problems will be presented. The generalization to 3 -D problems will be addressed in a forthcoming paper. The boundary $C$ and its portion $C_{0}$ are discretized into respectively $N$ and $M$ segments of equal length $h$. Let $C_{0}$ be centered at node $n$ of $C$ (Figure 2). The currents $\vec{J}_{2}$ and $\vec{M}_{2}$ are expanded along $C_{0}$ into $M$ pulse basis functions as

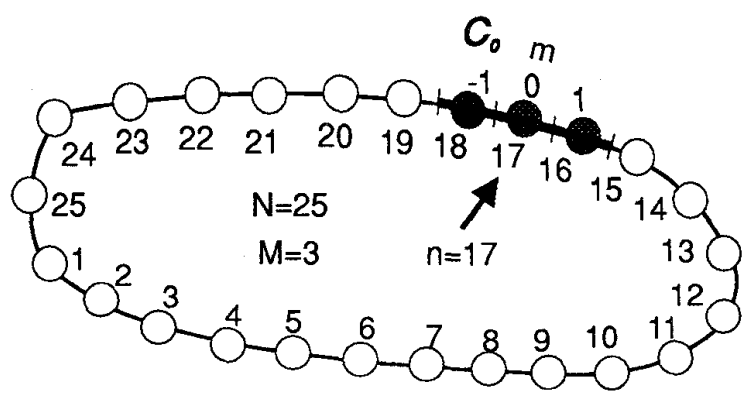

Fig. 2. Boundary meshing of the scatterer for IE-MEI.

$$
\begin{gathered}
\vec{J}_{2}(\ell)=\sum_{m=n-\frac{M-1}{2}}^{m=n+\frac{M-1}{2}} \vec{J}_{2}\left(\ell_{m}\right) \Pi\left(\ell-\ell_{m}\right), \\
\vec{M}_{2}(\ell)=\sum_{m=n-\frac{M-1}{2}}^{m=n+\frac{M-1}{2}} \vec{M}_{2}\left(\ell_{m}\right) \prod\left(\ell-\ell_{m}\right)
\end{gathered}
$$

where $0 \leq \ell \leq L$ is the arclength along the scatterer boundary and the pulse basis is

$$
\prod\left(\ell-\ell_{m}\right)=\left\{\begin{array}{l}
1 \text { if }\left|\ell-\ell_{m}\right|<h / 2 \\
0 \text { if }\left|\ell-\ell_{m}\right| \geq h / 2
\end{array}\right.
$$

Substituting (4) and (5) into equation (3) and interchanging the order of summation and integral operators, one obtains

$$
\begin{gathered}
\sum_{m=n-\frac{M-1}{2}}^{m=n+\frac{M-1}{2}}\left[\vec{J}_{2}\left(\ell_{m}\right) \cdot \int_{C_{\mathrm{o}}} \vec{E}_{t}^{s}(\ell) \prod\left(\ell-\ell_{m}\right) d \ell-\right. \\
\left.-\vec{M}_{2}\left(\ell_{m}\right) \cdot \int_{C_{0}} \vec{H}_{t}^{s}(\ell) \prod\left(\ell-\ell_{m}\right) d \ell\right]=\left\langle\vec{E}_{\text {null }}^{*}, \vec{J}_{s}\right\rangle
\end{gathered}
$$

Since $h$ is small and $\vec{E}^{s}$ is smooth along the PEC scatterer boundary, we can use $\vec{E}^{s}(\ell) \simeq \vec{E}^{s}\left(\ell_{m}\right)$. If the segments do not contain corners in the TM case, $\vec{H}^{s}$ is also smooth inside them and $\vec{H}^{s}(\ell) \simeq \vec{H}^{s}\left(\ell_{m}\right)$ for $\left|\ell-\ell_{m}\right|<h / 2$. If we define $\vec{a}_{n m}=h \vec{J}_{2}\left(\ell_{m}\right)$ and $\vec{b}_{n m}=h \vec{M}_{2}\left(\ell_{m}\right)$, we obtain

$\sum_{m=n-\frac{M-1}{2}}^{m=n+\frac{M-1}{2}}\left[\vec{a}_{n m} \cdot \vec{E}_{t}^{s}\left(\ell_{m}\right)-\vec{b}_{n m} \cdot \vec{H}_{t}^{s}\left(\ell_{m}\right)\right]=\left\langle\vec{E}_{\text {null }}^{*}, \vec{J}_{s}\right\rangle$

which is the discrete version of the IE-MEI.

The right-hand term in (7) is the residual $R=$ $\left\langle\vec{E}_{\text {null }}^{*}, \overrightarrow{J_{s}}\right\rangle$. The notation $\vec{E}_{\text {null }}$ has been previously used in [11], where the authors obtained the same residual for the finite difference MEI by using a different procedure. 
This residual will be zero for any possible incident field if the $\vec{E}_{\text {null }}$ function is orthogonal to the induced current $\vec{J}_{s}$. The physical interpretation of $\vec{E}_{\text {null }}$ is as follows:

- It is the electric field radiated along the object boundary by an array, placed in $C_{0}$, of $M$ electric point sources of strength $\vec{a}_{n m}$ and $M$ magnetic point sources of strength $\vec{b}_{n m}$

- If $\left|\vec{J}_{s}\right|=\delta\left(\vec{r}_{0}\right), R=\left\langle\vec{E}_{\text {null }}^{*}, \vec{J}_{s}\right\rangle=\vec{E}_{\text {null }}^{*}\left(\vec{r}_{0}\right)$ is the residual of the IE-MEI for a scattered field equal to the Green's function centered in $\vec{r}_{0}$, that is $\vec{E}^{s}(\vec{r})=\underline{G}\left(\vec{r}, \vec{r}_{0}\right) \cdot \vec{J}_{s}\left(\vec{r}_{0}\right)$ where $\underline{G}$ is the dyadic Green's function.

\section{Numerical solution for $\stackrel{2}{2} D$ problems}

If the residual of the IE-MEI (7) is zero, which can be achieved in the 2-D case for convex scatterer shapes with appropriate $\vec{a}_{n m}$ and $\vec{b}_{n m}$ coefficients [10], in two dimensions we have

$$
\sum_{m=n-\frac{M-1}{2}}^{m=n+\frac{M-1}{2}}\left[a_{n m} E_{t}^{s}\left(\ell_{m}\right)-b_{n m} H_{t}^{s}\left(\ell_{m}\right)\right]=0
$$

where

$$
\begin{gathered}
\text { for TM polarization } \\
a_{n m}=-\vec{a}_{n m} \cdot \hat{z}, \quad \vec{E}_{t}^{s}=-E_{t}^{s} \bar{z}, \\
b_{n m}=\vec{b}_{n m} \cdot \hat{\ell}, \quad \vec{H}_{t}^{s}=H_{t}^{s} \hat{\ell}
\end{gathered}
$$

for TE polarization

$$
\begin{aligned}
& a_{n m}=\vec{a}_{n m} \cdot \hat{\ell}, \quad \vec{E}_{t}^{s}=E_{t}^{s} \hat{\ell}, \\
& b_{n m}=\vec{b}_{n m} \cdot \hat{z}, \quad \vec{H}_{t}^{s}=H_{t}^{s} \hat{z}
\end{aligned}
$$

and $\hat{\ell}$ is the unit vector tangential to the scatterer boundary $C$. Equation (8) is a linear relation between the tangential components of the electric and magnetic fields at $M$ consecutive nodes in the portion $C_{0}$ of the scatterer boundary centered at node $n$, where $n=1 \ldots N$. This relation can be expressed more concisely as

$$
[A]\left[E_{t}^{s}\right]-[B]\left[H_{t}^{s}\right]=0
$$

where $a_{n m}$ are the elements of global matrix $[A], b_{n m}$ are the elements of global matrix $[B]$ and $E_{t}^{s}\left(\ell_{m}\right), H_{t}^{s}\left(\ell_{m}\right)$ are respectively the components of vectors $\left[E_{t}^{s}\right]$ and $\left[H_{t}^{s}\right]$. The matrices $[A]$ and $[B]$ are cyclic band diagonal with bandwidth $M$.

Since the tangential component of the electric scattered field is known along the object boundary, $\left[E_{t}^{s}\right]=-\left[E_{t}^{i}\right]$, we can obtain the tangential component of the magnetic scattered field $\left[H_{t}^{s}\right]$ as

$$
-[B]^{-1}[A]\left[E_{t}^{i}\right]=\left[H_{t}^{s}\right]
$$

and the electric induced current as

$$
J_{s}=H_{t}=H_{t}^{i}+H_{t}^{s}
$$

In order to compute the coefficients $a_{n m}$ and $b_{n m}$ we use the same procedure as in the finite difference formulation of the MEI [7] [8]. If in equation (7) the $\vec{E}_{\text {null }}$ function is orthogonal to the induced current $\vec{J}_{s}$, the residual is zero for all possible incident fields (the postulate of invariance of the MEI [7] [8]). This fact suggests that if we know for each mesh node $n P \geq 2 M-1$ linearly independent scattered field solutions (called "measuring functions") due to $P$ different incident fields, we can obtain a set of $2 M$ coefficients $a_{n m}, b_{n m}$ and these coefficients will also satisfy (8) with the actual scattered field. These measuring functions are obtained as the field radiated by a set of functions $\sigma_{p}\left(\vec{r}^{\prime}\right)$ defined on the scatterer boundary $C$, called "metrons".

In the TM case the electric and magnetic field components tangential to a smooth scatterer boundary $C$ radiated by current $J_{z}$ in an homogeneous medium are [2]

$$
\begin{aligned}
& E_{z}^{s}(\vec{\rho})=-\frac{k \eta}{4} \int_{C} J_{z}(\vec{\rho}) H_{0}^{(2}\left(k\left|\vec{\rho}-\vec{\rho}^{\prime}\right|\right) d \ell^{\prime} \\
& H_{\ell}^{s}(\vec{\rho})=\frac{J_{z}}{2}-\frac{1}{4 j} \int_{C} J_{z}\left(\vec{\rho}^{\prime}\right) \frac{\partial H_{0}^{(2}\left(k\left|\vec{\rho}-\vec{\rho}^{\prime}\right|\right)}{\partial n} d \ell^{\prime}
\end{aligned}
$$

while in the TE case these tangential field components are $[2]$

$$
\begin{gathered}
E_{\ell}^{s}(\vec{\rho})=-\frac{K \eta}{4} \int_{C}\left(\hat{n} \cdot \hat{n}^{\prime}\right) J_{\ell}\left(\vec{\rho}^{\prime}\right) H_{0}^{(2}\left(k\left|\vec{\rho}-\vec{\rho}^{\prime}\right|\right) d \ell^{\prime}- \\
-\frac{\eta}{4 k} \int_{C}\left[\frac{\partial}{\partial \ell^{\prime}} J_{\ell}(\vec{\rho})\right]\left[\frac{\partial}{\partial \ell} H_{0}^{(2}\left(k\left|\vec{\rho}-\vec{\rho}^{\prime}\right|\right)\right] d \ell^{\prime}
\end{gathered}
$$

$$
H_{z}^{s}(\vec{\rho})=-\frac{J_{\ell}}{2}-\frac{1}{4 j} \int_{C} J_{\ell}\left(\vec{\rho}^{\prime}\right) \frac{\partial H_{0}^{(2}\left(k\left|\vec{\rho}-\vec{\rho}^{\prime}\right|\right)}{\partial n^{\prime}} d \ell^{\prime}
$$

where $k$ is the wavenumber, $\eta$ the intrinsic impedance of the medium and $\hat{n}$ the unit normal to the boundary.

\section{Relation With FD-MEI}

According to K.Mei et al. [7] and R.Pous [8], in twodimensional problems, for each node in the truncation boundary $C$ of a finite difference mesh, there exists a linear relation involving the scattered field value $\phi^{s}\left(\vec{\rho}_{0}\right)$ at this node and the field $\phi^{s}\left(\vec{\rho}_{m}\right)$ at its $M-1$ neighbors (Figure 3), given as

$$
\sum_{m=0}^{M-1} c_{m} \phi^{s}\left(\vec{\rho}_{m}\right)=0
$$

where $\phi^{s}=E_{z}^{s}$ for TM polarization and $\phi^{s}=H_{z}^{s}$ for TE polarization. Since $H_{\ell}^{s} \propto \partial E_{z}^{s} / \partial n$ in the TM case and 


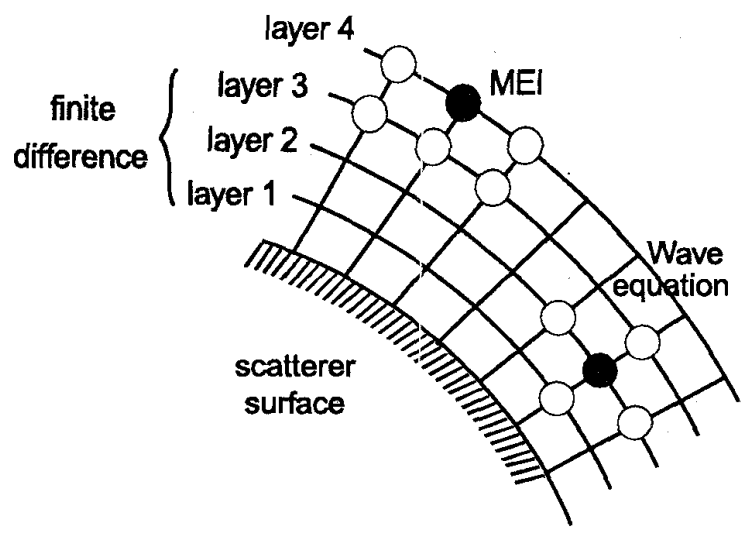

Fig. 3. Volume meshing of the scatterer for FD-MEI.

$E_{\ell}^{s} \propto \partial H_{z}^{s} / \partial n$ in the TE case, it is clear that the discrete IE-MEI (8) can be written as

$$
\sum_{m=n-\frac{M-1}{2}}^{m=n+\frac{M-1}{2}}\left[a_{n m} \phi^{s}\left(\ell_{m}\right)-b_{n m} \frac{\partial \phi^{s}\left(\ell_{m}\right)}{\partial n}\right]=0
$$

which is the same as the FD-MEI (17) if the normal derivative of $\phi^{s}$ is approximated by a finite difference.

The integral equation formulation of the MEI has four main advantages over the finite difference formulation:

- Easier meshing

- Smaller number of unknowns (same as FD-MEI with zero finite difference layers)

- There is no finite difference approximation. The normal derivative of the field is computed rigorously by the radiation operators $(13),(14),(15),(16)$.

- The linear system to be solved for $a_{n m}$ and $b_{n m}$ has a much better condition number than in the FD-MEI.

To conclude, the IE-MEI (8) is a boundary element method (BEM), since there are no finite-difference approximations and the coefficients $a_{n m}$ and $b_{n m}$ that relate tangential components of the electric and magnetic fields are obtained though the radiation operators (13), (14), (15),(16) with the appropriate Green's function. Furthermore, the original FD-MEI is a hybrid FD-BEM method in which an integral equation formulation is used to terminate the finite-difference MEI.

\section{NUMERICAL CONSIDERATIONS}

The following set of metrons has been used to obtain the results presented in this paper:

$$
\begin{array}{ll}
\sigma_{p}\left(\ell^{\prime}\right)=e^{j 2 \pi p \ell^{\prime} / L}, & |p| \leq \frac{P-1}{2}, \\
P=2 k R_{\max } \chi+1, & 0 \leq \ell^{\prime} \leq L
\end{array}
$$

which are harmonic functions along the scatterer boundary $C$ to order $P=k R_{\max } \chi$, where $R_{\max }$ is the radius of the smallest cylinder enclosing the scatterer and $\chi$ is a coefficient slightly larger than one. Typical values of $\chi$ range from 1.05 to 1.3 . To our experience, this set of metrons guarantees a small residual $R=\left\langle\vec{E}_{\text {null }}^{*}, \vec{J}_{s}\right\rangle$ for two-dimensional problems when the PEC scatterer is a convex cylinder [10].

For computation of the radiation operators (13), (14), $(15),(16)$, the metrons are discretized into pulse basis functions (5) with point-matching at the center of the pulses. One-point integration has been used over each basis function, except for self-impedance terms where the small argument approximation of the Green's function has been used. It must be noted that no significant improvement in our results was achieved with more integration points.

With regard to the number of nodes $M$ in the linear relation (8), the best results are obtained with $M \geq 5$, although the best trade-off between computation time and accuracy is achieved with $M=3$.

The memory requirement of our implementation of the IE-MEI in MATLAB 4.2 language is approximately $9.5 \mathrm{~N}$ complex numbers for a linear relation involving $M=3$ boundary nodes. This means that for each megabyte of available memory the IE-MEI method is able to allocate 6900 unknowns.

For each unknown at the mesh truncation boundary, the number of operations is proportional to $P$ times the number of integration points at the boundary, $Q$. In our case, $Q=N$. The total number of operations is thus proportional to $P Q N$, where $N$ is the number of nodes in the boundary of the grid, or the number of unknowns. Since the number of metrons $P$ in (19) increases linearly with the electrical size of the object, the total number of operations grows as the cube of the frequency: $P Q N \propto$ $f^{3}$. However, it is noted that the field due to the metrons (19) is a Fourier transform. For example, the electric field in the TM case (13) is

$$
E_{p}\left(\vec{\rho}_{m}\right)=-\frac{k \eta}{4} \int_{0}^{L} e^{-j 2 \pi p \ell^{\prime} / L} H_{0}^{(2}\left(k\left|\vec{\rho}_{m}-\vec{\rho}^{\prime}\right|\right) d \ell^{\prime}
$$

and similar relations exists for the TM magnetic field (14) and for the TE case (15), (16). Therefore, if the boundary samples are equally spaced, the FFT algorithm can be used and the total number of operations is in that case proportional to $N Q \log _{2} Q$, which grows with frequency as $f^{2} \log _{2} f$ instead of $f^{3}$.

Figure 4 shows the computer memory and CPU time required by the IE-MEI method compared with method of moments (MoM). For an honest comparison, the same discretization and computer code has been used in both methods to compute the radiation operators (13), (14), $(15),(16)$. 
Memory (Bytes)

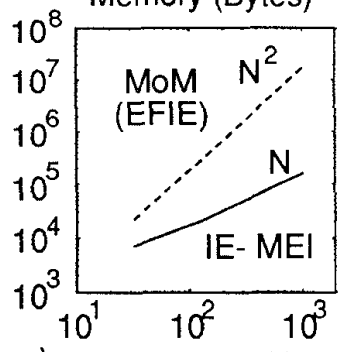

a) Unknowns $\mathrm{N}$
Total CPU time, sec.

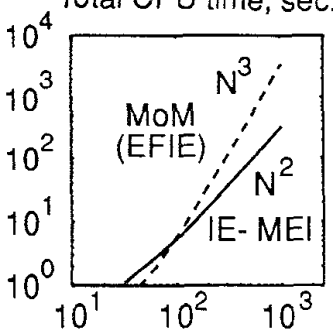

b) Unknowns $\mathrm{N}$

Fig. 4. Computer memory (a) and CPU time (b) requiered by IFMEI compared to BEM-MoM.

For all results presented in Figures 4 to 6 , the following parameters have been used:

- $M=3$ boundary nodes in the linear relation ( 8 )

- Number of unknowns $N$ equal to the smallest power of 2 that results in segments smaller than $0.15 \lambda$

- Number of metrons $P=N / 4$

- Total number of integration points $Q=N$

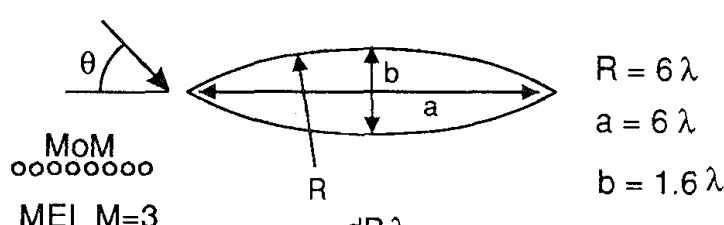

$\mathrm{dB} \lambda \quad \mathrm{MEI} M=3$

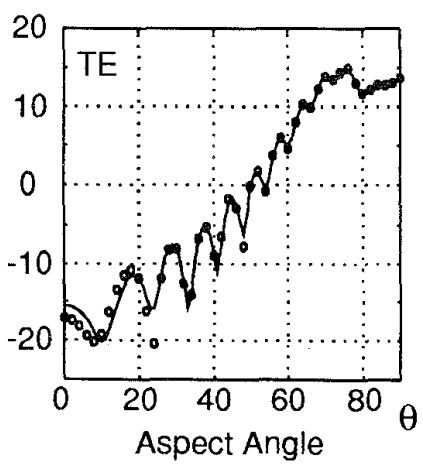

$\mathrm{dB} \lambda$

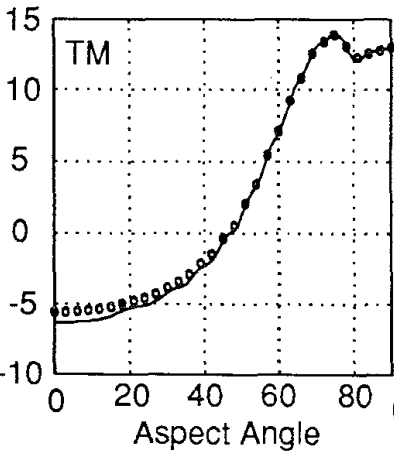

Fig. 5. Monostatic RCS of a resonant size ogive. IE-MEI results are compared to BEM-MoM.

\section{Results}

Figure 5 shows the monostatic RCS of an ogive $6 \lambda \times$ 1.6 $\lambda$. The results of the IE-MEI for the TM and TE polarizations are compared with the results of the $\mathrm{BE}-\mathrm{MoM}$ approach. The agreement is very good, and can still be improved for near edge incidences by setting $M>3$.

Figure 6 shows the monostatic RCS of the same ogive at a frequency 128 times larger. The electrical size of the ogive is now $768 \lambda \times 205 \lambda$ and the perimeter is $1620 \lambda$. This result was computed in a $486 \mathrm{DX}-66 \mathrm{PC}$ using only $2.3 \mathrm{MB}$ of memory. The results of the IE-MEI are compared to high frequency approximations: geometrical optics (GO) and geometrical theory of diffraction (GTD). Again, the agreement is excellent.

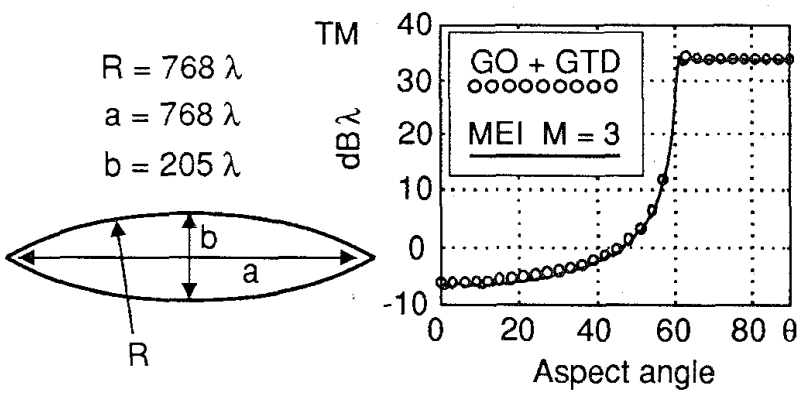

Fig. 6. Monostatic RCS of an electrically large ogive. IE-MEI results are compared to GO + GTD high-frequency methods.

\section{Conclusions}

A novel integral formulation of the measured equation of invariance (IE-MEI) has been derived from the reciprocity theorem and implemented for PEC 2-D scattering problems. It has been shown that the IE-MEI is equivalent to the original finite difference formulation (FD-MEI) [7] [8], which leads to the conclusion that the numerical procedure carried out in the FD-MEI to truncate a finite difference mesh is in fact a hybrid FD-BEM approach.

The resulting 2-D numerical algorithm for the IE-MEI uses sparse matrices, with computer memory requirement proportional to the number of unknowns $N$, in contrast $\theta$ with the conventional BE-MoM, which has $N^{2}$ memory requirement. A frequency dependent set of harmonic metrons has been used to guarantee good results with convex shapes [10]. Since the use of harmonic metrons leads to a Fourier transform formulation, the FFT algorithm can be used to achieve a total number of operations proportional to $f^{2} \log _{2} f$, while the total number of operations in the BE-MoM is proportional to $f^{3}$. The results obtained for $2-\mathrm{D}$ perfectly conducting scatterers agree very well with the BE-MoM for resonant size scatterers, or with the results of high frequency methods for electrically large objects. 


\section{ACKNOWLEDGMENT}

The authors are indebted to Prof. K. Michalski of Texas A\&M University for the valuable discussions on the MEI method.

\section{REFERENCES}

[1] Harrington, R. F., Field Computation by Moment Methods, MacMillan, New York, 1968

[2] Morita, N., Kumagai, N., Mautz, J.R., Integral Equation Methods for Electromagnetics, Artech House, 1990.

[3] Moore, T.G., Blaschack, J.G, Taflove, A., Kriegsmann, G.A, "Theory and Application of Radiation Boundary Operators", IEEE Trans. on Antennas and Propagation, 1988, 36, pp. $1797-1811$

[4] Kriegsmann, G.A, Taflove, A., Umashankar, K. R., "A New Formulation of Electromagnetic Wave Scattering Using an On-Surface Radiation Boundary Condition Approach", IEEE Trans. on Antennas and Propagation, 1987, 35, pp. 152-161

[5] Ramahi, O. M., Khebir, A., Mittra, R., "Numerically Derived Absorbing Boundary Condition for the Solution of Open Region Scattering Problems", IEEE Trans. on Antennas and Propagation, 1991, 39, pp. 350-353

[6] Jin, J.M., Liepa, V.V., "Application of Hybrid Finite Element Method to Electromagnetic Scattering from Coated Cylinders", IEEE Trans. on Antennas and Propagation, Vol. AP36 , pp. 50-54, January 1988.

[7] Mei, K. K., Pous, R., Chen, Z., Liu, Y.W., Prouty, M.D., "The Measured Equation of Invariance: a New Concept in Field Computations" IEEE Trans, on Antennas and Propagation, Vol. 42, no. 3, March 1994

[8] Pous, R., The Measured Equation of Invariance: a New Concept in Field Computation, Ph.D. dissertation, University of California at Berkeley, 1992

[9] Rius J.M., Parron J., Pous R., Cadama A., "The Measured Equation of Invariance: A Proof for the Postulates and a New Formulation as a Sparse Matrix Integral Equation Method", presented at 1994 URSI Radio Science Meeting, June 19-24, 1994, Seatle, Washington

[10] Rius, J.M., "On the Invariance of the MEI Method and the Choice of Metrons", submitted for publication to Microwave and Optical Technology Letters in July 1995

[11] Jevtic J.O., Lee R., "A Theoretical and Numerical Analysis of the Measured Equation of Invariance", IEEE Trans. on Antennas and Propagation, Vol. 42, No. 8, pp. 1097-1105, August 1994 